\title{
Joint Multichannel Motion Compensation Method for MIMO SAR 3D Imaging
}

\author{
Ze-min Yang, Meng-dao Xing, Guang-cai Sun, and Zheng Bao \\ Key Laboratory for Radar Signal Processing, Xidian University, Xian 710071, China \\ Correspondence should be addressed to Guang-cai Sun; rsandsgc@126.com
}

Received 26 January 2014; Revised 28 April 2014; Accepted 5 June 2014

Academic Editor: Wen-Qin Wang

Copyright ( 2015 Ze-min Yang et al. This is an open access article distributed under the Creative Commons Attribution License, which permits unrestricted use, distribution, and reproduction in any medium, provided the original work is properly cited.

\begin{abstract}
The multiple-input-multiple-output (MIMO) synthetic aperture radar (SAR) system with a linear antenna array can obtain 3D resolution. In practice, it suffers from both the translational motion errors and the rotational motion errors. Conventional singlechannel motion compensation methods could be used to compensate the motion errors channel by channel. However, this method might not be accurate enough for all the channels. What is more, the single-channel compensation may break the coherence among channels, which would cause defocusing and false targets. In this paper, both the translational motion errors and the rotational motion errors are discussed, and a joint multichannel motion compensation method is proposed for MIMO SAR 3D imaging. It is demonstrated through simulations that the proposed method exceeds the conventional methods in accuracy. And the final MIMO SAR 3D imaging simulation confirms the validity of the proposed algorithm.
\end{abstract}

\section{Introduction}

Conventional single-channel synthetic aperture radar (SAR) uses wideband signal and synthetic apertures to obtain high range resolution and high azimuth resolution, respectively. But it could not resolve along height due to the lack of baseline in the elevation direction. The imaging results are the projection from the scene in $3 \mathrm{D}$ to the range-azimuth plane. To resolve along height, the multiple-input-multipleoutput (MIMO) SAR system [1-3] uses multichannel to form the baseline in the third dimension. Technically, the MIMO SAR system could place its sensor in many different layouts. Two possible geometries of MIMO SAR are along-track array (for the reduction of azimuth-ambiguities, moving target indication, superresolution, etc.) and across-track array (for the reduction of elevation-ambiguities, interferometry, 3D imaging, etc.) [4].

The MIMO SAR system has two main advantages: (1) the degrees of freedom can be greatly increased by the concept of virtual array provided by the multiple antennas [3]; (2) the MIMO SAR system can provide plenty of transmitting elements and receiving elements to satisfy the cross-track sampling [5]. Due to the second advantage, it can significantly improve the resolution of the third dimension. Thus it is widely used for 3D imaging. Profiting from the unique advantages, airborne MIMO SAR 3D imaging technique has become a field of intensive research in recent years. However, studies about the MIMO SAR are mostly concentrated on antenna arrangements $[4,6]$, waveform designs $[7,8]$, and imaging algorithms $[4,9]$. The motion compensation (MOCO) has not been paid much attention. To deal with the motion errors, the most preferable way is using a highprecision navigation system. However, in many cases, due to the limit of accuracy of such systems, motion errors are unlikely to be compensated correctly. Thus MOCO methods based on raw data are widely used. Conventional MOCO methods [10-12] or autofocus methods [13,14] only deal with the translational motion errors of the carrier aircraft. This is reasonable in single-channel SAR imaging because the sensor is normally placed at the centroid of the plane. The rotational motion errors only alter the beam direction and could be compensated through beam control. Nevertheless, for the MIMO SAR system, the rotational motion errors could no longer be ignored because they affect the relative positions of the sensors. Conventionally the motion errors are estimated and compensated separately for each channel. We refer to 


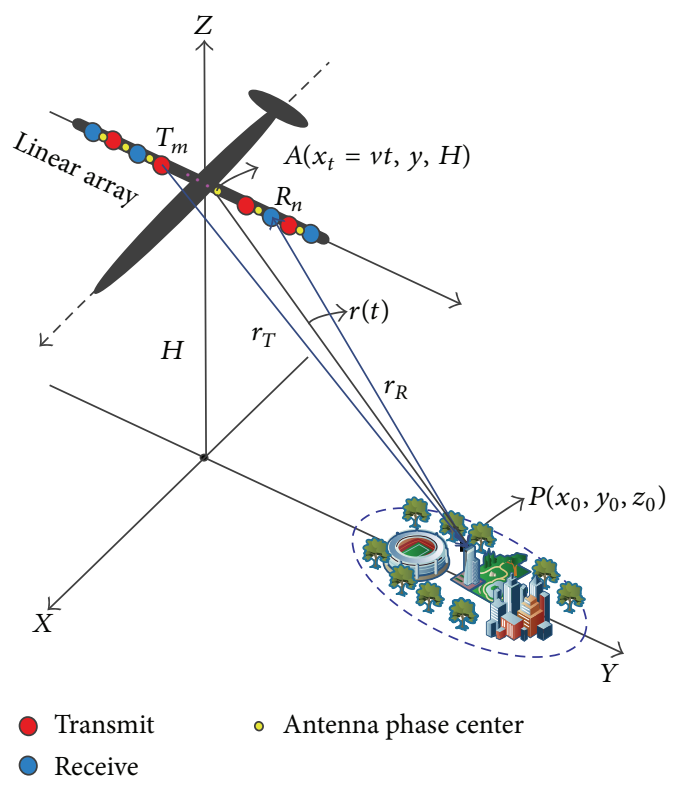

FIgURE 1: The ideal geometry of MIMO SAR 3D imaging.

this method as single-channel MOCO (SC-MOCO) method. This method may break the coherence among channels which would cause defocusing and false targets. In this paper, making use of the linear features of the MIMO system, a joint multichannel MOCO (JMC-MOCO) method is proposed for MIMO SAR 3D imaging. This new method can estimate and compensate both the translational motion errors and the rotational motion errors. Thus it is of high value for the MIMO SAR 3D imaging.

The paper is organized as follows. In Section 2, we first discuss the ideal geometry of MIMO SAR 3D imaging. Then both the translational motion errors and the rotational motion errors are analyzed in Section 3. In Section 4, the JMC-MOCO method for the MIMO SAR 3D imaging is introduced in detail. Simulations in Section 5 confirm that the proposed method is of higher accuracy than that of the conventional single-channel method.

\section{Signal Mode and Imaging Geometry of MIMO SAR}

The MIMO SAR utilizes an across-track array to gain the third dimension resolution. The transmitter and receiver antennas are usually distributed nonuniformly along the linear across-track array. Assume a MIMO SAR platform flies at the altitude of $H$ along the $X$-axis with velocity $v$. The MIMO array with $M$ transmit elements and $N$ receive elements is linearly laid out along the $Y$-axis. Signal sorting would be operated after the collecting of data, and $M N$ valuable signals can be saved for the imaging process during each pulse. The ideal geometry of MIMO SAR 3D imaging is indicated in Figure 1.

Ignoring two-way antenna characteristics and propagation attenuation, after demodulation and range compression, the echo signal transmitted by the $m$ th transmitter $T_{m}$ reflected by a generic point target $P\left(x_{0}, y_{0}, z_{0}\right)$ and received by the $n$th receiver $R_{n}$ is given by

$$
e(\tau, t)=\rho \cdot s\left(\tau-\frac{2 r(t)}{c}\right) \exp \left(-j 4 \pi \frac{r(t)}{\lambda}\right),
$$

where $s(\tau)$ is the transmitted signal, $\tau$ is the fast time, $t$ is the slow time, $\rho$ is the reflectivity of $P, c$ is the time speed, and $\lambda$ is the wavelength; $r(t)=\left(r_{T}+r_{R}\right) / 2$ [15] denotes the range from the antenna phase center (APC) $A\left(x_{t}=v t, y, H\right)$ of $T_{m}$ and $R_{n}$ to $P ; r_{T}$ and $r_{R}$ are the range from $T_{m}$ and $R_{n}$ to $P$, respectively. Then

$$
r(t)=\sqrt{\left(v t-x_{0}\right)^{2}+\left(y-y_{0}\right)^{2}+\left(H-z_{0}\right)^{2}},
$$

where $y$ is the across-track position of the APC.

As described in $[16,17]$, the image is formed in three steps, namely, the compression in the range direction, the focusing in the azimuth direction using the SAR principle, and the beam forming operation to focus the data in the across-track direction.

\section{Imaging Geometry of MIMO SAR with Motion Errors}

In practice, due to the presence of atmospheric turbulence that produces sensor track deviations from an ideal straight track, motion errors need to be accurately compensated. There have been numerous good methods dealing with the translational motion errors but ignoring the rotational motion errors [10-12]. These methods are adequate for singlechannel SAR, because the sensor is usually placed at the centroid of the carrier aircraft and the rotational motion errors can be compensated through beam control. However, for MIMO SAR system, the rotational motion errors would change the baseline position and decrease the quality of the final image eventually.

Before the introduction of our method, we first discuss the core element that causes the defocusing of SAR images, that is, the range migration error.

As shown in Figure 2, a blue dashed arrow identifies the offset from the ideal position of the carrier plane to its true position. Then the translational motion error is $\Delta \mathbf{r}=\left[\Delta x_{t}, \Delta y_{t}, \Delta z_{t}\right]$. The subscript $t$ means time-variant. In Figure 3 , the rotational motion error of the carrier aircraft is characterized by three angles $\left[\theta_{t}, \varphi_{t}, \phi_{t}\right]$ with $\theta_{t}$ denoting the pitch angle, $\varphi_{t}$ denoting the roll angle, and $\phi_{t}$ denoting the yaw angle. Among them, $\theta_{t}$ does not affect the array position. The rotation matrixes of $\varphi_{t}$ and $\phi_{t}$ can be written as $\mathbf{T}_{\varphi}=$ $\left[\begin{array}{ccc}1 & 0 & 0 \\ 0 & \cos \varphi_{t} & -\sin \varphi_{t} \\ 0 & \sin \varphi_{t} & \cos \varphi_{t}\end{array}\right]$ and $\mathbf{T}_{\phi}=\left[\begin{array}{ccc}\cos \phi_{t} & -\sin \phi_{t} & 0 \\ \sin \phi_{t} & \cos \phi_{t} & 0 \\ 0 & 0 & 1\end{array}\right]$ respectively. Then, the real position of the APC can be denoted as

$$
\begin{gathered}
\mathbf{A}=\left[x_{t}, 0, H\right]^{\mathrm{T}}+\mathbf{T}_{\boldsymbol{\varphi}} \mathbf{T}_{\phi}[0, y, 0]^{\mathrm{T}}+\Delta \mathbf{r}^{\mathrm{T}} \\
=\left[x_{t}-y \sin \phi_{t}+\Delta x_{t}, y \cos \phi_{t} \cos \varphi_{t}+\Delta y_{t},\right. \\
\left.\quad H+y \cos \phi_{t} \sin \varphi_{t}+\Delta z_{t}\right]^{\mathrm{T}} .
\end{gathered}
$$




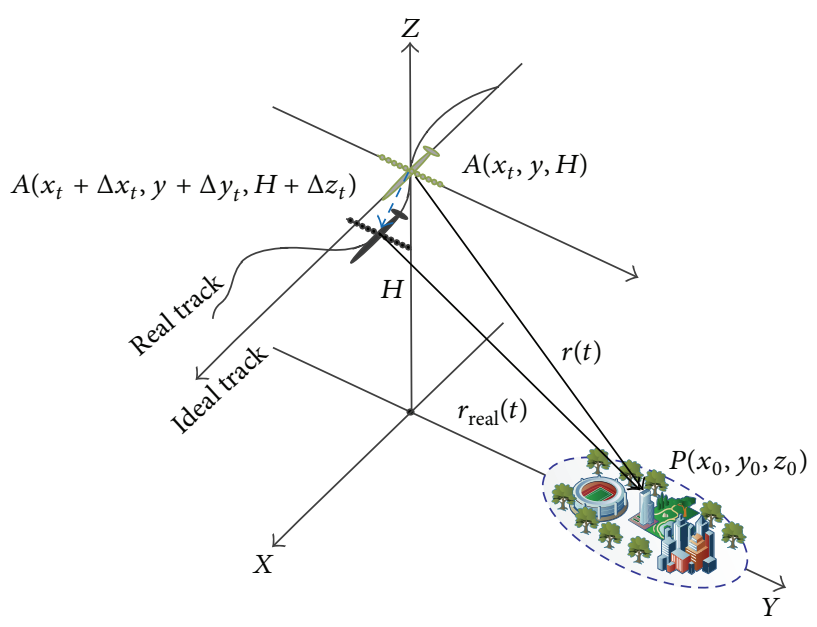

FIGURE 2: The geometry of MIMO SAR 3D imaging with translational motion errors.

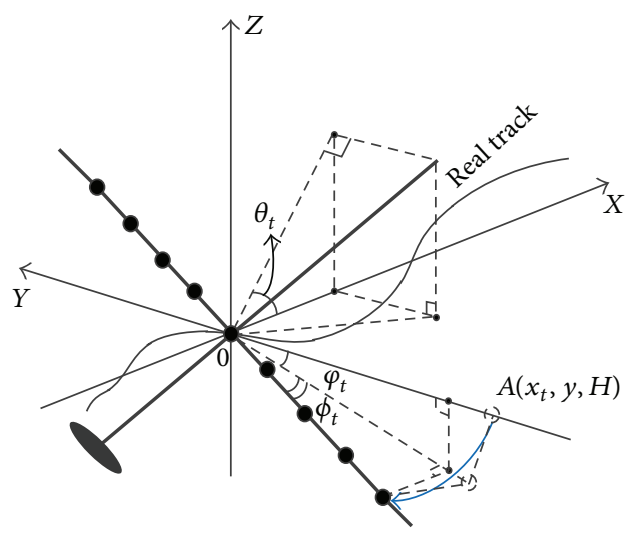

FIGURE 3: The geometry of MIMO SAR 3D imaging with rotational motion errors.

The slant range from $A$ to the point target $P$ can now be written as

$$
\begin{aligned}
r_{\text {real }}(t)= & \left(\left(x_{t}-y \sin \phi_{t}+\Delta x_{t}-x_{0}\right)^{2}\right. \\
& +\left(y \cos \phi_{t} \cos \varphi_{t}+\Delta y_{t}-y_{0}\right)^{2} \\
& \left.+\left(H+y \cos \phi_{t} \sin \varphi_{t}+\Delta z_{t}-z_{0}\right)^{2}\right)^{1 / 2} \\
= & \sqrt{r(t)^{2}+r_{e}^{2}},
\end{aligned}
$$

where

$$
\begin{aligned}
r_{e}=(2 & \left(x_{t}+\Delta x_{t}-x_{0}\right)\left(-y \sin \phi_{t}\right)+\Delta x_{t}^{2} \\
& +2 \Delta x_{t}\left(x_{t}-x_{0}\right)+2\left(-\Delta y_{t} y_{0}-\Delta z_{t} z_{0}\right) \\
& +2 y\left(y_{0}\left(1-\cos \phi_{t} \cos \varphi_{t}\right)-z_{0} \cos \phi_{t} \sin \varphi_{t}\right. \\
& \left.\left.\quad+\Delta y_{t} \cos \phi_{t} \cos \varphi_{t}+\Delta z_{t} \cos \phi_{t} \sin \varphi_{t}\right)\right)^{1 / 2}
\end{aligned}
$$

is the range error caused by the translational and rotational motion errors and needs to be compensated. In the next
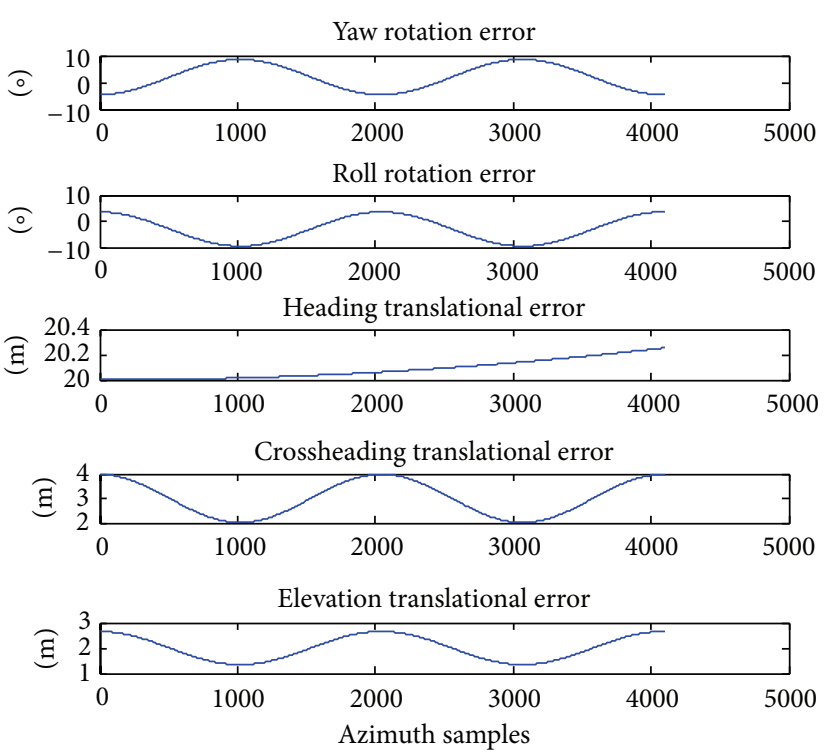

Figure 4: The simulated rotational motion errors and the translational motion errors.

section, the JMC-MOCO method is discussed in detail to estimate and compensate the range error.

\section{Joint Multichannel MOCO Method for MIMO SAR 3D Imaging}

To compensate the motion errors, the conventional method is used to estimate and compensate the motion errors for each channel. However, the inevitable estimating errors of each channel may cause the incoherence among channels which would cause defocusing and false targets. Combining the linear properties of the across-track array, in this section we present a new method which can compensate both the translational motion errors and the rotational motion errors and preserve the coherence among different channels at the same time.

To avoid the incoherence after motion error compensation, the motion error estimating and compensating need to be jointed among channels. From (4) and (5) we can see that, due to the rotational motion errors, the range error differs along the linear across-track array. To clearly uncover the relationship between the range error and the array, we expand (4) according to Taylor expansion as follows:

$$
r_{\text {real }}(t) \approx r_{B}+\frac{\left(x_{t}-x_{0}\right)^{2}}{2 r_{0}}+\alpha_{t}+\beta_{t} \cdot y+\frac{y^{2}}{2 r_{0}},
$$

where $r_{0}=\sqrt{y_{0}^{2}+\left(H-z_{0}\right)^{2}}$ is the ideal zero-Doppler range from the center APC to the point target, $\left(x_{t}-x_{0}\right)^{2} / 2 r_{0}$ is the ideal range migration, and

$$
\begin{aligned}
\alpha_{t}= & \frac{\Delta x_{t}\left(x_{t}-x_{0}\right)}{r_{0}}-\Delta y_{t} \frac{y_{0}}{r_{0}}+\Delta z_{t} \frac{H-z_{0}}{r_{0}} \\
& +\frac{\Delta x_{t}^{2}+\Delta y_{t}^{2}+\Delta z_{t}^{2}}{2 r_{0}}
\end{aligned}
$$




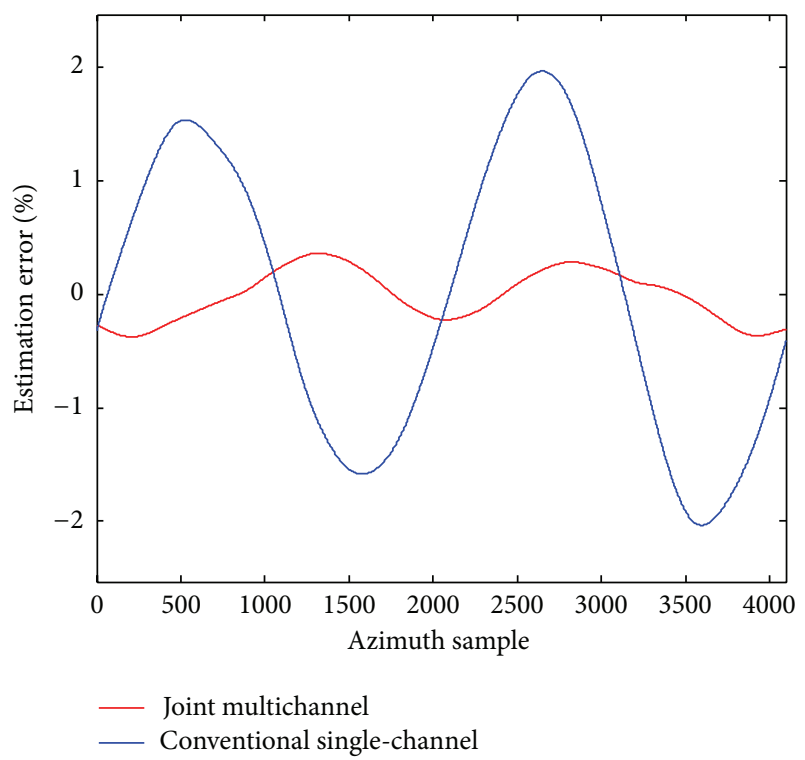

Figure 5: Comparison of the center APC Doppler rate estimation accuracy between the proposed method and the conventional method.

$$
\begin{aligned}
\beta_{t}= & -\frac{x_{t}-x_{0}}{r_{0}} \sin \phi_{t}-\frac{y_{0}}{r_{0}} \cos \phi_{t} \cos \varphi_{t} \\
& +\frac{H-z_{0}}{r_{0}} \cos \phi_{t} \sin \varphi_{t} .
\end{aligned}
$$

$y^{2} / 2 R_{B}$ is typically small and can be neglected. Then the range error $\alpha_{t}+\beta_{t} \cdot y$ is linear along the across-track array. Moreover, $\alpha_{t}$ is only related to the translational motion errors, and $\beta_{t}$ is only related to the rotational motion errors.

The range error alters the Doppler rate and finally defocuses the image. Hence, to estimate the motion error, let us first analyze the true Doppler rates. As expressed in (1), after range compression, the phase history of $P$ is

$$
\Phi(t)=-\frac{4 \pi r_{\text {real }}(t)}{\lambda}
$$

Substitute (6) into (8) and derive (8) twice, and the Doppler rate can be denoted as

$$
\gamma=\gamma_{0}+A(t)+B(t) y
$$

where

$$
\gamma_{0}=-\frac{2 v^{2}}{\left(\lambda R_{B}\right)}
$$

is the ideal Doppler rate,

$$
\begin{gathered}
A(t)=\left(-2\left[\Delta a_{x t}\left(x_{t}-x_{0}+\Delta x_{t}\right)+2 \Delta v_{x t} v+\Delta v_{x t}^{2}\right.\right. \\
\left.\left.-\Delta a_{y t} y_{0}+\Delta a_{z t}\left(H-z_{0}\right)\right]\right)\left(\lambda r_{0}\right)^{-1}, \\
B(t)=\frac{2}{\lambda r_{0}}\left[2 v \omega_{\phi t} \cos \phi_{t}+a_{\phi t}\left(x_{t}-x_{0}\right) \cos \phi_{t}\right. \\
\left.-\omega_{\phi t}^{2}\left(x_{t}-x_{0}\right) \sin \phi_{t}\right] \\
+\frac{2 y_{0}}{\lambda r_{0}}\left[-a_{\phi t} \sin \phi_{t} \cos \varphi_{t}-a_{\varphi t} \cos \phi_{t} \sin \varphi_{t}\right. \\
+2 \omega_{\varphi t} \omega_{\phi t} \sin \phi_{t} \sin \varphi_{t} \\
\left.-\left(\omega_{\varphi t}^{2}+\omega_{\phi t}^{2}\right) \cos \phi_{t} \cos \varphi_{t}\right] \\
+\frac{2\left(H-z_{0}\right)}{\lambda r_{0}}\left[a_{\phi t} \sin \phi_{t} \sin \varphi_{t}-a_{\varphi t} \cos \phi_{t} \cos \varphi_{t}\right. \\
+2 \omega_{\phi t} \omega_{\varphi t} \sin \phi_{t} \cos \varphi_{t} \\
\left.+\left(\omega_{\phi t}^{2}+\omega_{\varphi t}^{2}\right) \cos \phi_{t} \sin \varphi_{t}\right] .
\end{gathered}
$$

In (11), $\Delta v_{x t}$ is the speed error of the plane. $\Delta a_{x t}, \Delta a_{y t}$, and $\Delta a_{z t}$ denote the translational acceleration in the track direction, the across-track direction, and the elevation direction, respectively. $\omega_{\phi_{t}}$ and $\omega_{\varphi_{t}}$ denote the angular velocity of the yaw angle and the roll angle. $a_{\phi}$ and $a_{\varphi}$ are the corresponding angular acceleration.

From (9) we can see that the true Doppler rate $\gamma$ contains three parts: the ideal Doppler rate $\gamma_{0}$ and two error terms. Similar to the case of range, $A(t)$ is only related to the translational motion errors, and $B(t)$ is only related to the rotational motion errors. That means, due to the rotational motion errors, the true Doppler rate scales linearly with the across-track position of the APC. The SC-MOCO method does not estimate the linear property of the motion errors; consequently it breaks the coherence among channels after motion compensation. This linear feature is used in the following to design a new motion compensation method that compensates both the translational motion errors and the rotational motion errors.

Suppose there are $K$ channels. Using the map drift (MD) method $[18,19]$ to estimate the Doppler rate of each channel, we have the Doppler rate array

$$
\widehat{\gamma}=\left[\widehat{\gamma}_{1}, \widehat{\gamma}_{2}, \ldots, \widehat{\gamma}_{k}, \gamma_{K}\right]^{\mathrm{T}}
$$

where $\widehat{\gamma}_{k}$ denotes the Doppler rate of the $k$ th channel. In order to estimate the two unknown parameters $A$ and $B$ in (9), a cost function is designed as follows:

$$
J(A, B)=\sum_{k=1}^{K}\left(\widehat{\gamma}_{k}-\gamma_{0}-A-B y_{k}\right)^{2},
$$




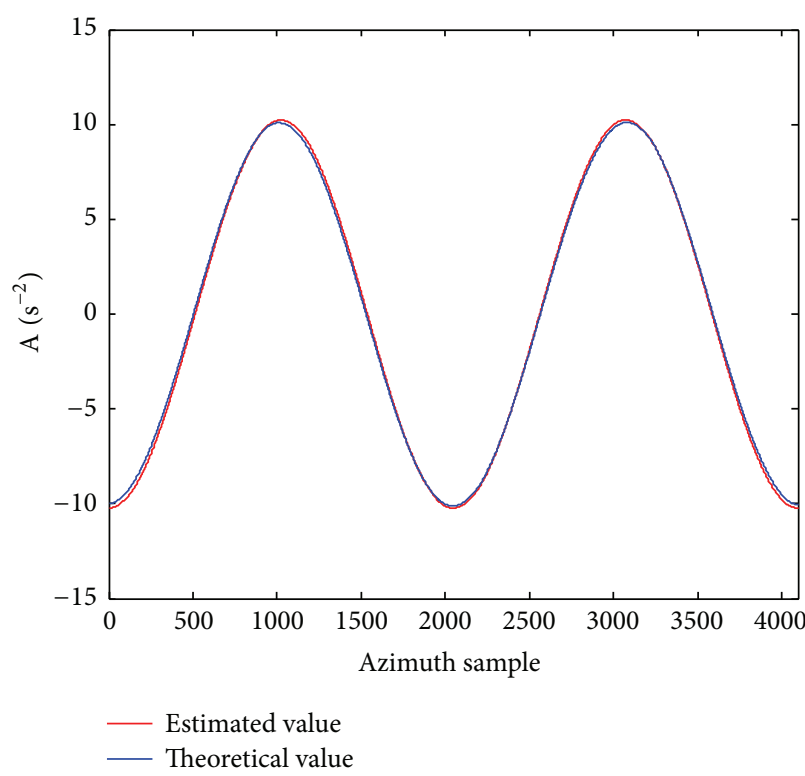

(a)

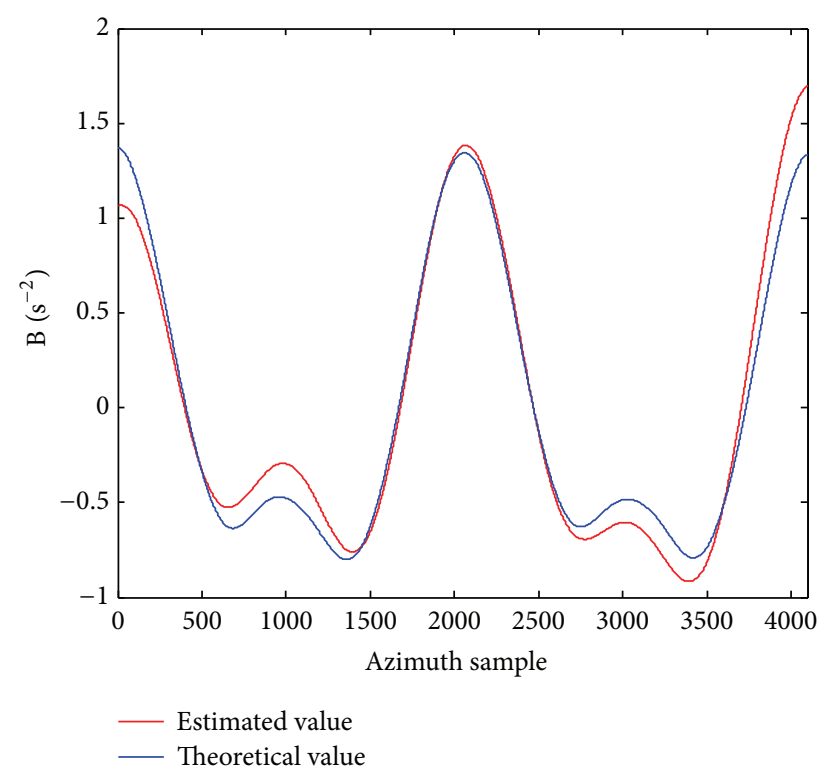

(b)

FIgURE 6: Comparison between the estimated value and the theoretical value of (a) $A$ and (b) $B$.

where $y_{k}$ is the position of the $k$ th channel. The aim is to find $(A, B)$ that minimizes $J$, that is, the stagnation point. Let the partial derivatives of $J$ be equal to 0 ; we have

$$
\begin{gathered}
\frac{\partial J}{\partial A}=-2 \sum_{k=1}^{K} \widehat{\gamma}_{k}+2 K \gamma_{0}+2 K A+2 B \sum_{k=1}^{K} y_{k}=0 \\
\frac{\partial J}{\partial B}=-2 \sum_{k=1}^{K}\left(\widehat{\gamma}_{k} y_{k}\right)+2 \gamma_{0} \sum_{k=1}^{K} y_{k} \\
+2 A \sum_{k=1}^{K} y_{k}+2 B \sum_{k=1}^{K} y_{k}^{2}=0 .
\end{gathered}
$$

By solving (14), $A$ and $B$ can be written as

$$
\begin{gathered}
A=\frac{\sum_{k=1}^{K} \widehat{\gamma}_{k} \times \sum_{k=1}^{K} y_{k}^{2}-\sum_{k=1}^{K}\left(\widehat{\gamma}_{k} y_{k}\right) \times \sum_{k=1}^{K} y_{k}}{N \sum_{k=1}^{K} y_{k}^{2}-\left(\sum_{k=1}^{K} y_{k}\right)^{2}}-\gamma_{0}, \\
B=\frac{K \sum_{k=1}^{K}\left(\widehat{\gamma}_{k} y_{k}\right)-\sum_{k=1}^{K} \widehat{\gamma}_{k} \times \sum_{k=1}^{K} y_{k}}{K \sum_{k=1}^{K} y_{k}^{2}-\left(\sum_{k=1}^{K} y_{k}\right)^{2}} .
\end{gathered}
$$

This method combines all the estimating results of each channel to obtain the linear coefficient and then derives each Doppler rate. Comparing to the conventional SC-MOCO method, this method has three advantages: (1) the estimating accuracy is dramatically improved; (2) the influence from occasional bad estimating values can be suppressed; and (3) the multichannels maintain coherence after motion estimation. This will be demonstrated in Section 5 through simulations.

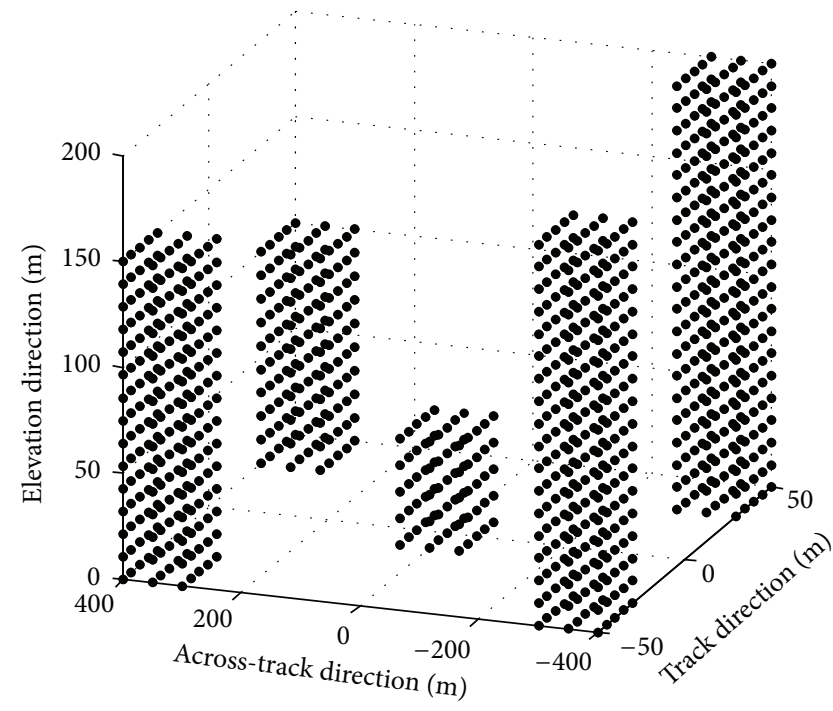

Figure 7: Point targets layout.

\section{Simulations}

5.1. Motion Error Estimation Comparison between the Proposed Method and the Conventional Method. In order to prove that the proposed method is of higher precision than the conventional method, a MIMO SAR system with a linear MIMO array is simulated. The key simulation parameters are listed in Table 1 . The linear MIMO array contains 32 APCs. Nine points are set in a line along the track direction with spacing of $2 \mathrm{~m}$. Common translational motion errors and rotational motion errors shown in Figure 4 are imported 


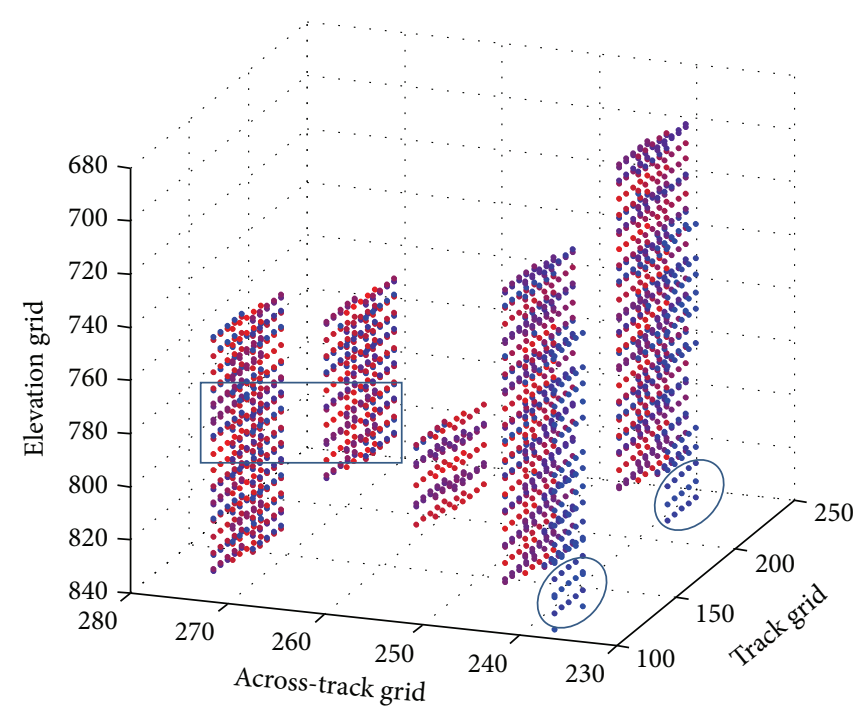

(a)

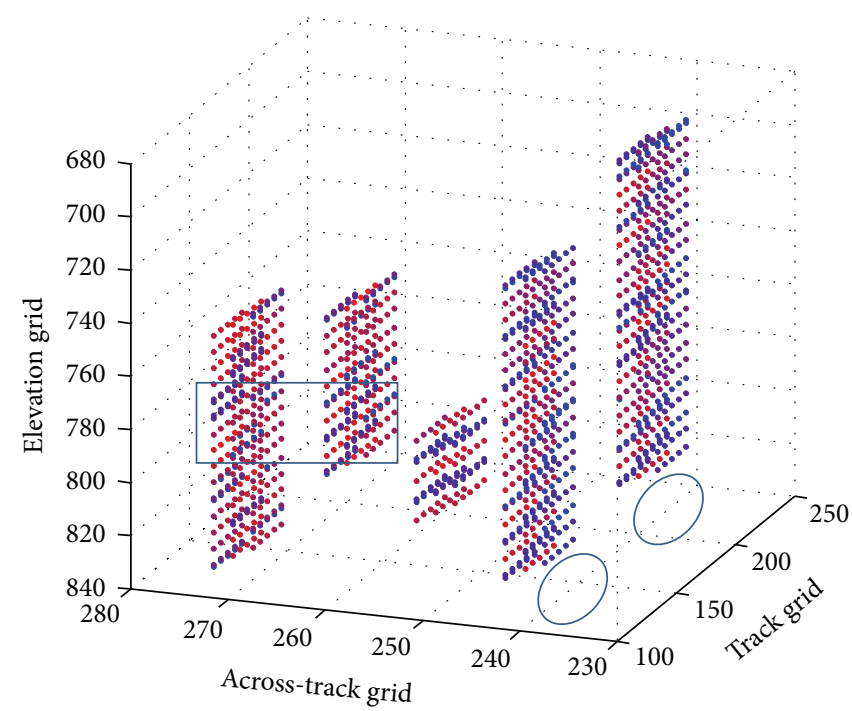

(b)

FIGURE 8: The imaging results with (a) the SC-MOCO method and (b) the proposed JMC-MOCO method.

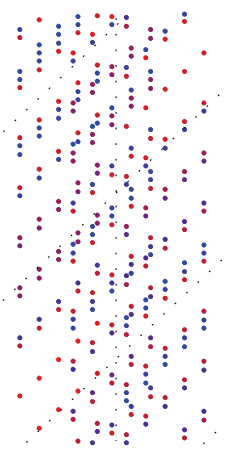

(a)

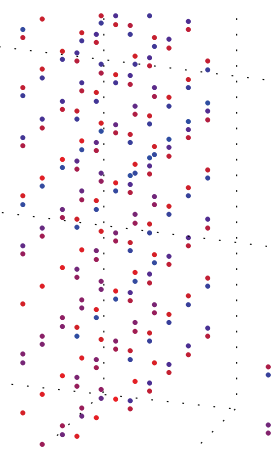

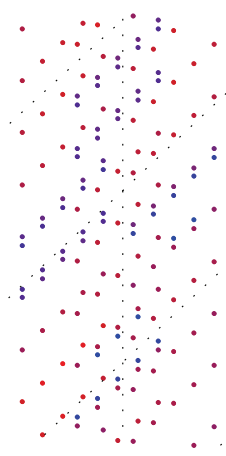

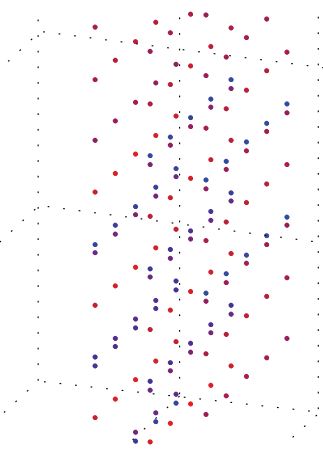

(b)

FIGURE 9: The magnified imaging results with (a) the SC-MOCO method and (b) the proposed JMC-MOCO method.

TABLE 1: Simulation parameters.

\begin{tabular}{lc}
\hline Wavelength & $0.0313 \mathrm{~m}$ \\
Bandwidth & $150 \mathrm{MHz}$ \\
Pulse duration & $2 \mu \mathrm{s}$ \\
PRF & $180 \mathrm{~Hz}$ \\
Antenna width & $2 \mathrm{~m}$ \\
Flying height & $6 \mathrm{~km}$ \\
Flying speed & $150 \mathrm{~m} / \mathrm{s}$ \\
Incidence angle & $60^{\circ}$ \\
Baseline length & $8 \mathrm{~m}$ \\
\hline
\end{tabular}

into the simulation. As shown in Figure 4, the yaw angle and the roll angle vary in a range of $\pm 10^{\circ}$. The translational motion errors in the across-track direction and the elevation direction are within $\pm 4 \mathrm{~m}$. All the motion errors mentioned above vary frequently. The translational motion error in the track direction is, nonetheless, quite different due to the enormous inertia of the plane. It is hardly time-varying but accumulative. Hence, the added translational motion error in the track direction is around $20 \mathrm{~m}$ and changes slowly.

After range compression and migration correction, the echo data are processed separately by the proposed JMCMOCO method and the conventional SC-MOCO method.

For the center APC, the Doppler rate estimation errors (defined as Estimation error $=$ ((Estimated value Real value)/Real value) $\times 100 \%$ ) of these two methods are shown in Figure 6. As can be seen from Figure 5, the Doppler rate estimation errors of the proposed method are small and less undulate, which means that the proposed method has significant improvement in estimation accuracy. Figure 6 shows the estimation results and the real value of the Doppler rate coefficients $A$ and $B$. Figures 5 and 6 prove that the proposed method is of high accuracy.

5.2. MIMO SAR 3D Imaging Simulation. Now we apply our method to simulated MIMO SAR 3D imaging and compare the imaging results with the conventional method. In the simulation, a complex 3D model containing 5 tall "buildings" 
is constructed with 1020 points. The structure of the model is illustrated in Figure 7. The simulated linear MIMO array contains 512 equivalent APCs with spacing of $\lambda / 2$, where $\lambda$ is the wavelength. The motion errors in Figure 4 are imported into the simulation. The parameters in Table 1 are adopted.

After MOCO with the conventional method and the proposed JMC-MOCO method, the 3D imaging results are shown in Figures 8(a) and 8(b), respectively. Only the points whose energy is stronger than $-10 \mathrm{~dB}$ of the strongest points are pointed in Figure 8 in different colors according to their energy. As we can see, the 5 "buildings" in Figure 8(b) are finely focused, while, in Figure 8(a), there are some unexpected false points, as marked in circles. This is because the respective estimation and compensation of each channel break the incoherence. This problem is well fixed in the proposed JMC-MOCO method. The subscene marked in a box in Figure 8 is magnified in Figure 9 for better comparison. As can be seen, the $3 \mathrm{D}$ model is better focused with the proposed method. Hence, the proposed MOCO method can significantly improve the imaging quality.

\section{Conclusions}

This paper proposed a JMC-MOCO method to estimate and compensate both the translational motion errors and the rotational motion errors for the MIMO SAR 3D imaging. Instead of estimating and compensating motion errors channel by channel, the proposed MOCO method utilizes the linear properties of the linear MIMO array, combining all the channel data to improve the accuracy of motion error estimation. It is demonstrated through $3 \mathrm{D}$ scenario imaging simulation that this new method can significantly improve the image quality.

\section{Conflict of Interests}

The authors declare that there is no conflict of interests regarding the publication of this paper.

\section{References}

[1] X. Zhuge and A. G. Yarovoy, "A sparse aperture MIMO-SARbased UWB imaging system for concealed weapon detection," IEEE Transactions on Geoscience and Remote Sensing, vol. 49, no. 1, pp. 509-518, 2011.

[2] Y. Yang and R. S. Blum, "MIMO radar waveform design based on mutual information and minimum mean-square error estimation," IEEE Transactions on Aerospace and Electronic Systems, vol. 43, no. 1, pp. 330-343, 2007.

[3] W. Q. Wang, "Virtual antenna array analysis for MIMO synthetic aperture radars," International Journal of Antennas and Propagation, vol. 2012, Article ID 587276, 10 pages, 2012.

[4] J. H. G. Ender and J. Klare, "System architectures and algorithms for radar imaging by MIMO-SAR," in Proceedings of the IEEE Radar Conference (RADAR '09), pp. 1-6, Pasadena, Calif, USA, May 2009.

[5] W. Wang, "MIMO SAR imaging: Potential and challenges," IEEE Aerospace and Electronic Systems Magazine, vol. 28, no. 8, pp. 18-23, 2013.
[6] X. Wencheng, Z. Xiaoling, and S. Jun, "MIMO antenna array design for airborne down-looking 3D imaging SAR," in Proceedings of the 2nd International Conference on Signal Processing Systems (ICSPS '10), pp. V2452-V2456, Dalian, China, July 2010.

[7] W. Wang, "Space-time coding MIMO-OFDM SAR for highresolution imaging," IEEE Transactions on Geoscience and Remote Sensing, vol. 49, no. 8, pp. 3094-3104, 2011.

[8] G. Krieger, M. Younis, S. Huber et al., "MIMO-SAR and the orthogonality confusion," in Proceeding of the 32nd IEEE International Geoscience and Remote Sensing Symposium (IGARSS '12), pp. 1533-1536, Munich, Germany, July 2012.

[9] Z. B. Wu, Y. T. Zhu, and Y. Su, "Spectral domain filling and 3D SAR imaging of airborne MIMO array," in Proceedings of the IEEE Region 10 Conference (TENCON '13), pp. 1-4, Xi'an, China, October 2013.

[10] A. Moreira and Y. Huang, "Airborne SAR processing of highly squinted data using a chirp scaling approach with integrated motion compensation," IEEE Transactions on Geoscience and Remote Sensing, vol. 32, no. 5, pp. 1029-1040, 1994.

[11] P. Prats, K. A. C. De Macedo, A. Reigber, R. Scheiber, and J. J. Mallorqui, "Comparison of topography- and aperturedependent motion compensation algorithms for airborne SAR," IEEE Geoscience and Remote Sensing Letters, vol. 4, no. 3, pp. 349-353, 2007.

[12] J. C. Kirk Jr., "Motion compensation for synthetic aperture radar," IEEE Transactions on Aerospace and Electronic Systems, no. 3, pp. 338-348, 1975.

[13] W. Ye and T. S. Yeo, "Weighted least-squares estimation of phase errors for SAR/ISAR autofocus," IEEE Transactions on Geoscience and Remote Sensing, vol. 37, no. 5, pp. 2487-2494, 1999.

[14] G. A. Bendor and T. W. Gedra, "Single-pass fine-resolution SAR autofocus," in Proceedings of the IEEE National Aerospace and Electronics Conference (NAECON '83), pp. 482-488, Dayton, Ohio, USA, May 1983.

[15] X. M. Peng, Y. P. Wang, W. X. Tan, W. Hong, and Y. Wu, "Downward looking 3D SAR based on uniform virtual phase centre restricted symmetrical distributed thinned array," in Proceedings of the 6th International Conference on Radar (RADAR '11), pp. 380-383, Chengdu, China, October 2011.

[16] J. Klare, "A new airborne radar for 3D imaging-simulation study of ARTINO," in Proceedings of the 6th European Conference on Synthetic Aperture Radar (EUSAR '06), Dresden, Germany, May 2006.

[17] J. Klare, A. Brenner, and J. Ender, "A new airborne radar for 3D imaging-image formation using the ARTINO principle," in Proceedings of the 6th European Conference on Synthetic Aperture Radar (EUSAR '06), Dresden, Germany, May 2006.

[18] C. E. Mancill and J. M. Swiger, "A map drift autofocus technique for correcting higher order SAR phase errors," in Proceedings of the 27th Annual Tri-Service Radar Symposium Record, pp. 523525, 1981.

[19] P. Samczynski and K. S. Kulpa, "Coherent mapdrift technique," IEEE Transactions on Geoscience and Remote Sensing, vol. 48, no. 3, pp. 1505-1517, 2010. 

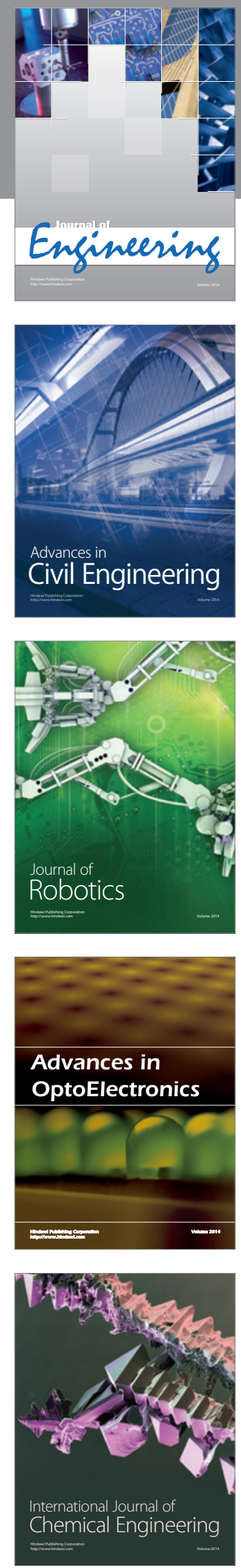

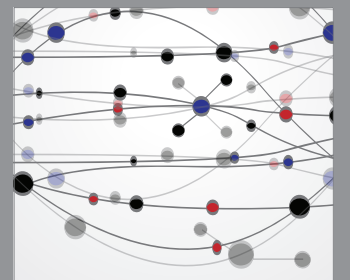

The Scientific World Journal
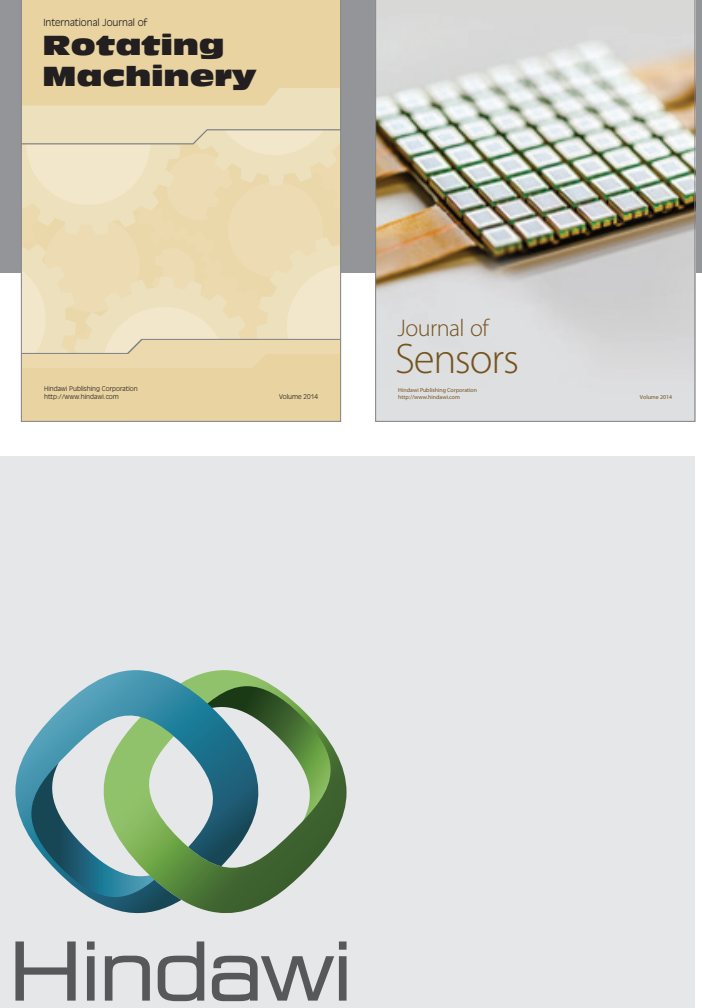

Submit your manuscripts at http://www.hindawi.com
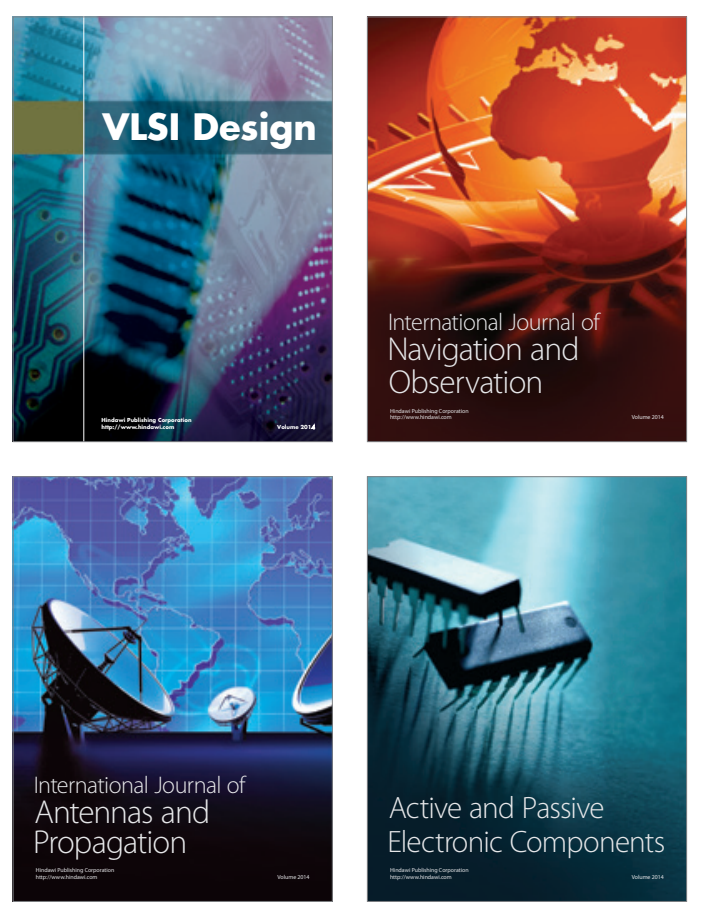
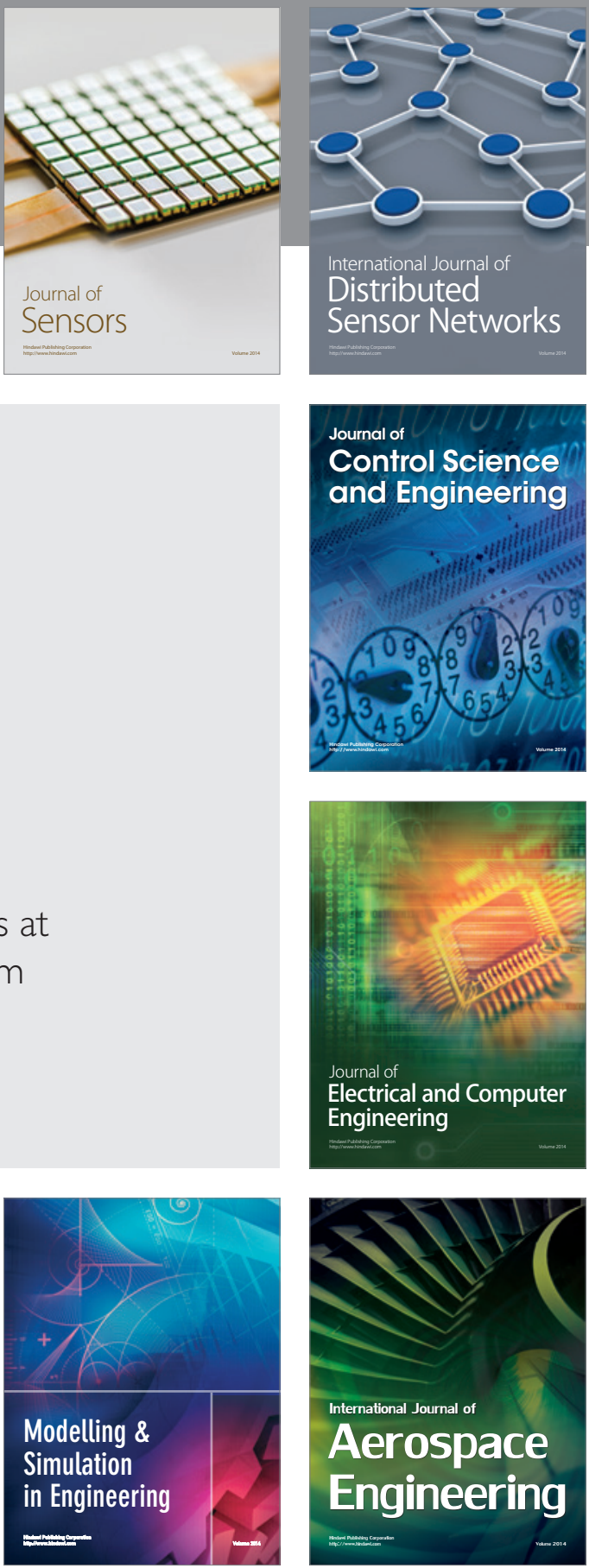

Journal of

Control Science

and Engineering
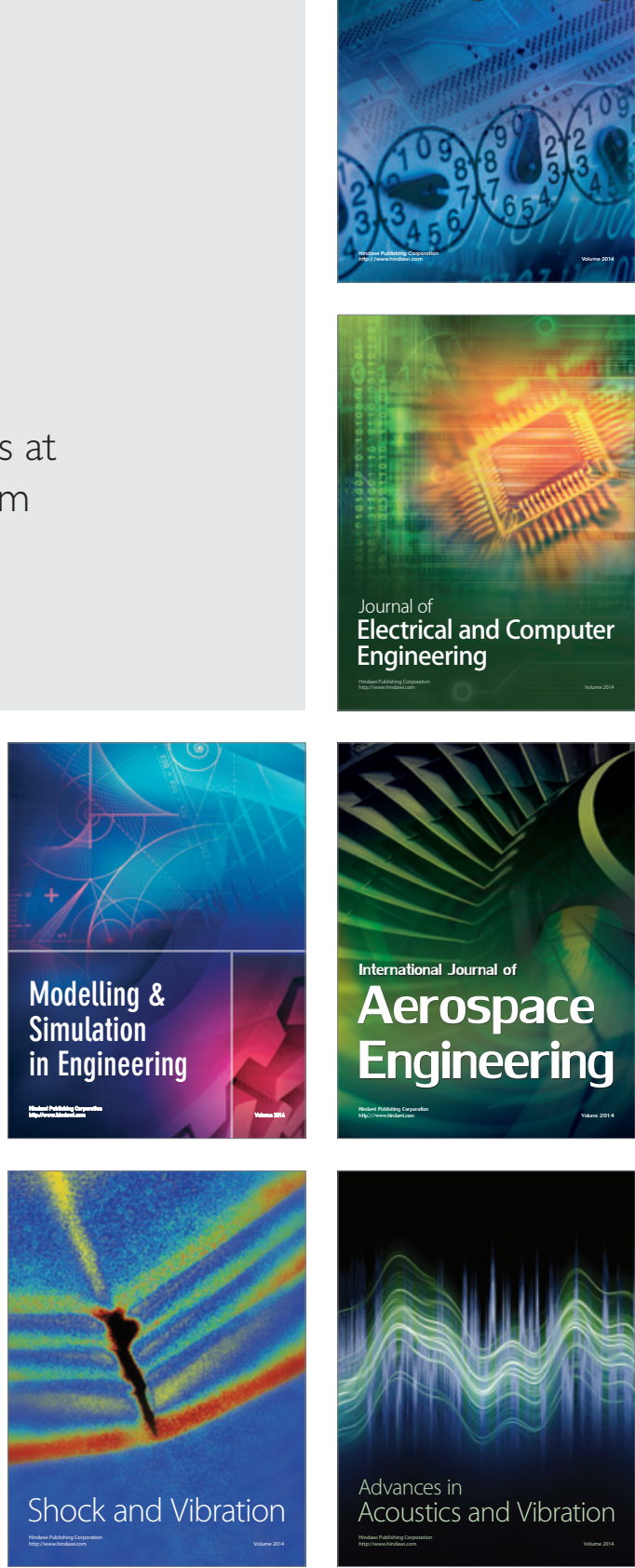